\title{
增材制造压电陶瓷研究进展
}

南 博 ${ }^{1,2}$, 藏佳栋 ${ }^{3}$, 陆文龙 ${ }^{3}$, 杨廷旺 ${ }^{3}$, 张升伟 ${ }^{3}$, 张海波 ${ }^{1,2}$

(1. 华中科技大学 材料科学与工程学院, 材料成形与模具技术国家重点实验室, 武汉 430074; 2. 广东华中科技大 学工业技术研究院，东莞 523808; 3. 深圳市基克纳科技有限公司，深圳 518100)

摘 要: 压电陶瓷是一种可以实现机械信号和电信号相互转换的功能陶瓷。由压电陶瓷与有机相构成的复合材料具 有不同的宏观连接方式, 这不仅决定了压电器件广泛的应用场合, 而且推动了压电陶瓷材料和器件多样化的成型 技术发展。与传统成型技术相比，增材制造技术的最大优势在于无需模具即可实现外形复杂的小批量样品快速成型， 这与多样化的压电陶瓷及其器件研发需求十分契合，同时因其样品后续加工量少、原材料利用率高、无需切削液的 特点, 得到了学术界和工业界的广泛关注。在陶瓷材料增材制造领域, 功能陶瓷和器件的研究仍在增长期。本文从 不同增材制造技术角度，探讨和对比现阶段无铅和含铅压电陶瓷增材制造的发展历史、原料制备、外形设计、功能 特性检测及试样的应用，并根据现阶段各增材制造技术的优、劣势对其未来进行了展望。

关 键 词：增材制造；含铅压电陶瓷；无铅压电陶瓷；功能特性；综述

中图分类号: TM282 文献标志码: A

\section{Recent Progress on Additive Manufacturing of Piezoelectric Ceramics}

\author{
NAN Bo ${ }^{1,2}$, ZANG Jiadong $^{3}$, LU Wenlong ${ }^{3}$, YANG Tingwang ${ }^{3}$, ZHANG Shengwei ${ }^{3}$, ZHANG Haibo $^{1,2}$ \\ (1. State Key Laboratory of Materials Processing and Die \& Mould Technology, School of Materials Science and Engineering, \\ Huazhong University of Science and Technology, Wuhan 430074, China; 2. Guangdong HUST Industrial Technology Research \\ Institute, Dongguan 523808, China; 3. Shenzhen Geekvape Technology Co., Ltd., Shenzhen 518100, China)
}

\begin{abstract}
Piezoelectric ceramic is a type of functional ceramic, which is able to convert the mechanical signal and the electronic signal mutually. Composed of piezoelectric ceramics and organic phase, piezoelectric composites have different kinds of connectivities, which not only determine the diverse applications of piezoelectric devices, but also promote the development of various shaping techniques in manufacturing piezoelectric materials and devices. In comparison with the traditional shaping methods, the most distinguishable advantage of additive manufacturing lies in its ability of quickly shaping a small batch of samples into geometrically complex designs without a mould, which makes it a highly suitable technique for investigating piezoelectric ceramics and its derivative devices in different kinds of connectivities. Meanwhile, the final additively manufactured samples require only tiny post-processing, have a high rate of utilization of the raw material and do not need cutting fluid during manufacturing. Due to the above-mentioned advantages, it attracts the widespread concerns from both
\end{abstract}

收稿日期: 2021-06-07; 收到修改稿日期：2021-08-18; 网络出版日期：2021-11-01

基金项目: 广东华中科技大学工业技术研究院广东省制造装备数字化重点实验室(2020B1212060014); 东莞市引进创新科 研团队计划(2020607101007)

Guangdong HUST Industrial Technology Research Institute, Guangdong Provincial Key Laboratory of Digital Manufacturing Equipment (2020B1212060014); Dongguan Innovative Research Team Program (2020607101007)

作者简介：南 博(1989-), 男，博士. E-mail: bonan@hust.edu.cn NAN Bo (1989-), male, PhD. E-mail: bonan@hust.edu.cn

通信作者: 张海波，教授. E-mail: hbzhang@hust.edu.cn ZHANG Haibo, professor. E-mail: hbzhang@hust.edu.cn 
academic and industrial communities. When focusing in the field of additive manufacturing ceramics, the data of scientific reports in additive of manufacturing functional ceramics and devices prove that it is still in a growing period. In the perspective of different additive manufacturing techniques, this article discusses and compares additive manufacturing of both lead-free and lead-based piezoelectric ceramics in the aspects of their historical development of each technique, preparation of the raw materials, geometrical designs, measurement of functional properties, and applications of the printed samples, and forecasts the future development based on the current benefits and drawbacks of each additive manufacturing technique.

Key words: additive manufacturing; lead-based piezoceramics; lead-free piezoceramics; functional property; review

压电陶瓷是一种广泛应用于微电子、汽车、自 动化、医疗影像、军事等领域的电子陶瓷 ${ }^{[1-7]}$ 。目前, 商业化的压电陶瓷以含铅体系为主, 具有良好的综 合性能。然而, 铅对环境有害且不可生物降解, 会随 着水循环和大气循环进入人体 ${ }^{[8-9]}$ 。近年来, 制备可 替代含铅体系的无铅压电陶瓷和器件成为学术界和 工业界的重要议题。

压电陶瓷的材料成分固然重要, 在很大程度上 决定了其功能性能, 然而压电陶瓷在某一领域的应 用还由压电陶瓷的宏观连接方式(Connectivity)决 定。连接方式的概念于 1978 年由 Newnham 等 ${ }^{[10]}$ 提出, 当时科学家正在设计适当的压电陶瓷传感器 结构, 以提高由陶瓷和高分子材料组成的复合材料 的品质因数, 最大程度发挥二者的压电特性和柔性,
提出了连接方式的概念。对于由两相构成的复合物, 连接方式共有 10 种, 即 $0-0 、 1-0 、 2-0 、 3-0 、 1-1$ 、 2-1、3-1、2-2、3-2 和 3-3, 如图 1(a)所示。多样化 的连接方式也推动了成型技术的发展。例如, 针对 2-2 连接方式(用于电容、储能、压电县臂梁等), 压 电陶瓷膜的膜厚逐渐变薄, 这为流延成型 ${ }^{[11]}$ 、旋转 涂布 ${ }^{[12]}$ 的研究带来机遇; 针对 1-3 连接方式(用于超 声换能器、声呐等), 不同精度呈阵列状分布的压电 陶瓷可以通过机械切削 ${ }^{[13]}$ 、凝胶注模 ${ }^{[14]}$ 、喷墨打 印 $^{[15]}$ 等技术制备。成型技术是连接压电陶瓷和压电 器件的纽带。

增材制造(Additive Manufacturing, AM)近年来 受到广泛关注。目前, 高分子材料增材制造技术已 逐渐成熟, 金属增材制造生产的零部件也已进入汽 (a)

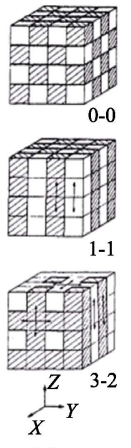

(d)
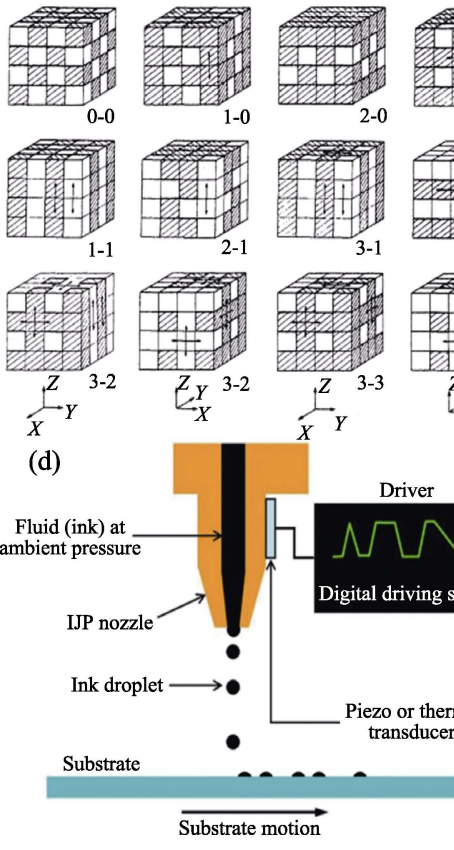
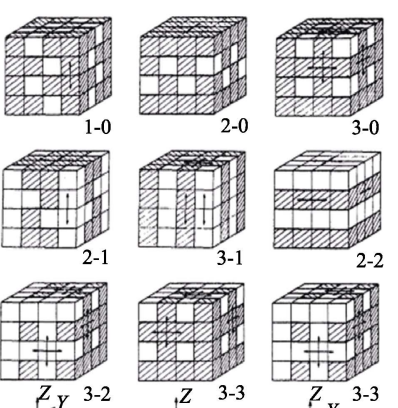

$$
\text { -2 }
$$

(e)

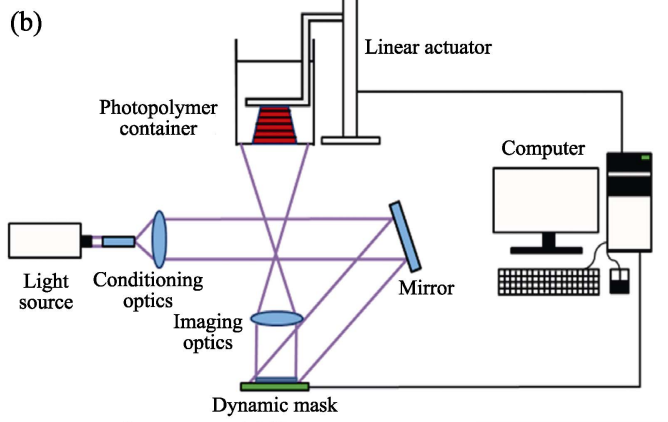

(b)
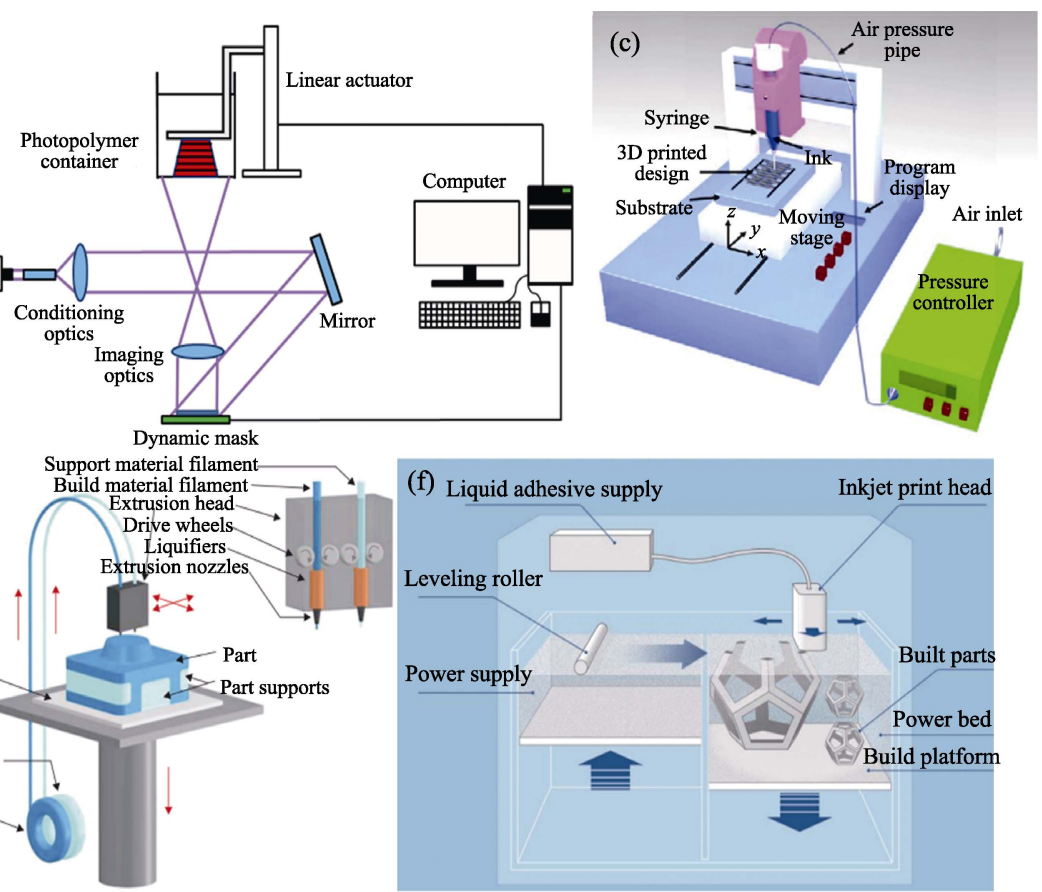

图 1 压电陶瓷的连接方式与常用于压电陶瓷的增材制造技术示意图

Fig. 1 Connectivities of piezoelectric ceramics and schematic pictures of common AM techniques applied for preparing piezoelectric ceramics

(a) 10 types of connectivitities in bi-phase composites ${ }^{[10]}$; (b) Vat photopolymerization ${ }^{[18]}$; (c) Direct ink writing ${ }^{[21]}$; (d) Inkjet printing ${ }^{[23]}$; (e) Fused deposition modelling ${ }^{[26]}$; (f) Binder jetting ${ }^{[29]}$ 
表 1 陶瓷增材制造技术的优缺点和原材料成分对比

Table 1 Comparison of advantages and disadvantages and composition of the feedstocks among several AM techniques applied in ceramics

\begin{tabular}{|c|c|c|c|c|c|}
\hline AM techniques & Advantages & Disadvantages & Ingredients of raw materials & Binder system & Ref. \\
\hline $\begin{array}{l}\text { Vat photo- } \\
\text { polymerization (VP) }\end{array}$ & $\begin{array}{l}\text { Low surface roughness, } \\
\text { high printing accuracy }\end{array}$ & $\begin{array}{l}\text { High cost of ceramic } \\
\text { paste and machine, } \\
\text { low degree of open } \\
\text { source }\end{array}$ & $\begin{array}{l}\text { Photosensitive polymer }+ \\
\text { ceramic powder/ceramic } \\
\text { precursor }\end{array}$ & $\begin{array}{l}\text { Photosensitive } \\
\text { polymer }\end{array}$ & [18-20] \\
\hline $\begin{array}{l}\text { Direct ink writing } \\
\text { (DIW) }\end{array}$ & $\begin{array}{l}\text { Open source, } \\
\text { multi-materials printing }\end{array}$ & $\begin{array}{l}\text { Clogging of nozzles, } \\
\text { high surface } \\
\text { roughness }\end{array}$ & $\begin{array}{l}\text { Powder }+ \text { polymer solution } \\
\text { (high viscosity)/ceramic } \\
\text { precursor }\end{array}$ & Water/oil based & [21- 22] \\
\hline Inkjet printing (IP) & $\begin{array}{l}\text { Open source, high } \\
\text { printing accuracy }\end{array}$ & $\begin{array}{l}\text { Low solids loading, } \\
\text { easy precipitation of } \\
\text { the particles }\end{array}$ & $\begin{array}{l}\text { Powder }+ \text { polymer solution } \\
\text { (low viscosity)/ceramic } \\
\text { precursor }\end{array}$ & Water/oil based & [23-25] \\
\hline $\begin{array}{l}\text { Fused deposition } \\
\text { modelling (FDM) }\end{array}$ & $\begin{array}{l}\text { Open source, low cost } \\
\text { of the machine }\end{array}$ & $\begin{array}{l}\text { Low relative density, } \\
\text { low accuracy }\end{array}$ & $\begin{array}{l}\text { Ceramic powder }+ \text { polymer } \\
\text { (filament) }\end{array}$ & $\begin{array}{l}\text { Thermal plastic } \\
\text { polymers }\end{array}$ & [26-28] \\
\hline Binder jetting $(\mathrm{BJ})$ & $\begin{array}{l}\text { High quality, gradient } \\
\text { materials }\end{array}$ & $\begin{array}{l}\text { High cost of machine, } \\
\text { reuse of the powder } \\
\text { bed }\end{array}$ & $\begin{array}{l}\text { Powder bed }+ \text { polymer } \\
\text { solution }\end{array}$ & Water/oil based & [29] \\
\hline
\end{tabular}

车行业 ${ }^{[16-17]}$, 而陶瓷和玻璃的增材制造技术发展缓 慢，仍未实现产业化应用。这是由陶瓷和玻璃材料 的本征特性决定的：一是因为陶瓷熔点高，导热性 能逊于金属，难以使用激光熔融技术进行成型加工; 二是因为陶瓷和玻璃常温塑性、韧性差，必须借助 高分子聚合物提高可塑性，进而改善其可加工性 能。表 1 列举了目前常用的陶瓷材料增材制造技术 的优缺点和原材料成分。与传统加工方式不同, 每 种增材制造技术都有难点和优势。粉末床需要的原 料量大、成本高, 而压电陶瓷粉末的材料种类多、 可购买的标准牌号有限, 不适合实验室研究, 这也 是粉末床类压电陶瓷增材制造报道较少的原因之 一。无论光固化还是直写式等需要浆料的增材制造 技术，都具有原料用量少，粉末的理化性能和浆料 的固含量、均匀性可调的优点, 故目前报道较多。

图 1(b f)为常用压电陶瓷增材制造的设备示意图。 目前，压电陶瓷增材制造的文献主要集中在成型工 艺参数和功能性能的检测, 偶见与器件相关的报 道。本文从不同增材制造技术角度阐述压电陶瓷增 材制造的历史、材料体系、功能性能及应用场景, 并 对其未来可能的研究方向进行展望。

\section{1 光固化成型}

光固化技术可追溯至 18 世纪，而现代光固化技 术与增材制造的结合起源于 20 世纪 70 年代 ${ }^{[30]}$ 。 1971 年, Swainson ${ }^{[31]}$ 提出用两束相交的放射性光线 在材料中引发高分子聚合物交联或者降解建立三维
物体。由于当时设备的限制，该方法的制备精度低 而被迫中止。之后, Hull 等 ${ }^{[32-33]}$ 在此基础上建立了 现代光固化成型技术的构想，即逐层曝光固化成型 三维物体。随着高分子和曝光技术的发展，在光敏 高分子材料中添加陶瓷粉末制备可光固化的陶瓷浆 料，实现陶瓷材料的光固化成型。近年来，光敏陶瓷 前驱体与光固化技术结合，成为光固化陶瓷增材制 造的重要研究方向之一 ${ }^{[34-35] 。}$

压电陶瓷立体光固化技术成型始于本世纪初。 法国研究人员 ${ }^{[36-38]}$ 率先报道了 PZT 的制作过程, 他 们从配制光固化浆料入手, 详细阐述了黏结剂、固 含量、表面活性剂等因素对浆料流变学的影响, 结 合曝光固化工艺阐述生坏制备工艺，并在此基础上 对试样的小型化进行了探讨。随着压电陶瓷的无铅 化趋势, 研究人员开始运用立体光固化技术制备无 铅压电陶瓷。Kim 等 ${ }^{[39}$ 运用水热反应制备 $\mathrm{BaTiO}_{3}(\mathrm{BT})$ 纳米粉末, 表面修饰后与聚乙二醇二丙 烯酸酯和 2,2-二羟甲基丙酸混合，通过控制光辐射 功率、单体浓度、光引发剂浓度和粉末固含量, 对 打印工艺进行优化，实现快速 $(<2 s)$ 打印。然后他们 利用不同形状的掩模，制备了 BT 的点阵列、正方形 阵列和蜂窝状阵列, 如图 2 所示。Chen 等 ${ }^{[40]}$ 采用丁 酮(体积分数 $66 \%$ )和乙醇(体积分数 $34 \%$ )混合溶剂 作为分散体系，将 BT 粉末和分散剂与之混合，对 BT 粉末进行表面修饰。经表面修饰的 BT 粉末和光 敏树脂混合得到光敏浆料, 用以制备柱状阵列的样 品。经 $1330{ }^{\circ} \mathrm{C}$ 烧结的样品进行介电测试, 在 $1 \mathrm{kHz}$ 频率下的介电常数 $\left(\varepsilon_{\mathrm{r}}\right)$ 为 1300 , 介电损耗 $(\tan \delta)$ 仅为 

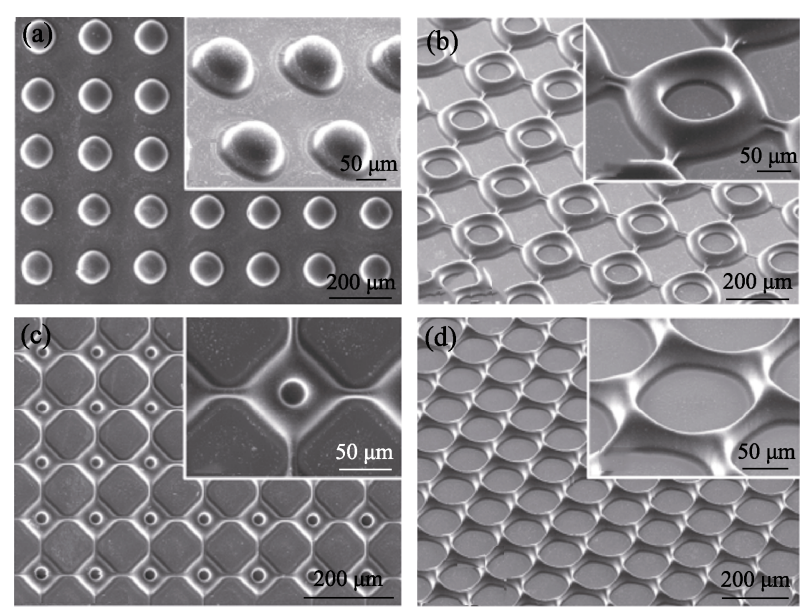

图 2 不同形状的压电陶瓷 BT 阵列 ${ }^{[39]}$

Fig. 2 Piezoelectric BT arrays with different shapes ${ }^{[39]}$

(a) Dot array; (b, c) Square arrays with different sized void spaces; (d) Honeycomb array

0.012 。在 $100{ }^{\circ} \mathrm{C}$ 、电场 $30 \mathrm{kV} / \mathrm{cm}$ 极化 $30 \mathrm{~min}$ 后, 压 电常数 $d_{33}$ 为 $160 \mathrm{pC} / \mathrm{N}$ 。将光固化技术制备的 $\mathrm{BT}$ 试样性能与传统固相反应法制备的 BT 性能(介电常 数为 1700 , 介电损耗 $<0.1$, 压电常数 $d_{33}$ 为 $190 \mathrm{pC} / \mathrm{N}$ ) 进行对比发现 ${ }^{[41]}$, 两种技术制备的 BT 性能相近。
之后, Song 等 ${ }^{[19]}$ 在文献[40]的工作基础上, 对立体 光固化技术制得的试样封装制成超声传感器。经过 测试, 在 2.35 2.8 $\mu \mathrm{s}$ 范围内可以清晰地看到强度为 $(0.11 \pm 0.055) \mathrm{V}$ 的回声响应, 这说明超声传感器可以 有效地实现超声信号和电信号转换。除了 $\mathrm{BT}$ 基压 电陶瓷，立体光固化技术还应用于无铅压电陶瓷 $\mathrm{KNaNbO}_{3}(\mathrm{KNN})$ 的制备。Chen 等 ${ }^{[42]}$ 通过微型立体光 固化技术(Micro-stereolithography)制备 KNN 环形阵 列, 样品居里温度为 $230{ }^{\circ} \mathrm{C}$, 介电常数为 2150 , 介 电损耗为 0.08 , 在室温、电场 $3 \mathrm{kV} / \mathrm{mm}$ 条件下对样 品进行极化, 压电常数 $d_{33}$ 为 $170 \mathrm{pC} / \mathrm{N}$ 。这与传统固 相反应法制得的 $\mathrm{KNN}$ (居里温度为 $230{ }^{\circ} \mathrm{C}$, 压电常 数 $d_{33}$ 为 $300 \mathrm{pC} / \mathrm{N}$ )相比 ${ }^{[43]}$, 性能略有下降。在结构 设计方面, Cui 等 ${ }^{[44]}$ 利用立体光固化技术实现高固 含量浆料成型，同时将超材料对三维结构的节点单 元应用在压电陶瓷微观设计上(如图 3 所示), 使样 品在微观上获得更多可能性的连接方式。通过高精 度的微纳结构设计结构单元, 实现材料高自由度设计 的机电耦合各向异性和顺向效应，这为今后触觉传 感、源头检测、声学传感等压电器件指明了研究方向。

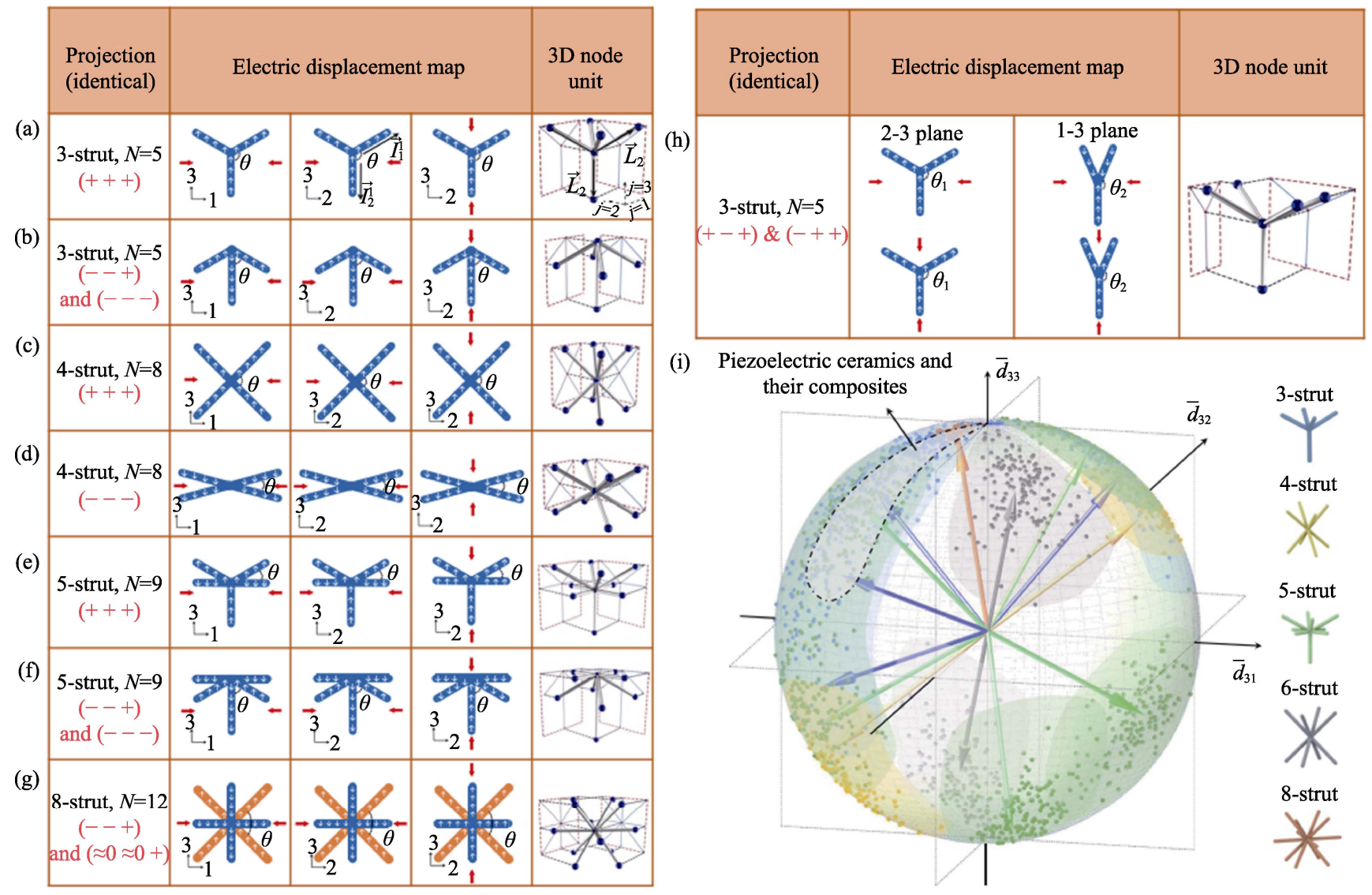

图 3 可调节压电常数的压电超材料设计 ${ }^{[44]}$

Fig. 3 Design of piezoelectric metamaterials for tailorable piezoelectric charge constants ${ }^{[44]}$ (a-g): Node unit designs from 3-, 4-, 5- and 8-strut identical projection patterns, respectively; (h) Node unit with dissimilar projection patterns showing decoupled $\bar{d}_{31}, \bar{d}_{32}$; (i) Dimensionless piezoelectric anisotropy design space accommodating 
除了无铅压电陶瓷成型，立体光固化技术还可 用于制备含铅压电超声传感器。Chen 等 ${ }^{[45]}$ 运用立体 光固化技术将不同固含量的浆料(质量分数 $78.0 \%$ 、 $80.0 \% 、 81.8 \%$ 和 $89.0 \%$ )分别成型, 研究发现固含量 为质量分数 $81.8 \%$ 的浆料制得的样品性能最优, 其 介电常数为 765 , 介电损耗为 0.020 , 在 $70{ }^{\circ} \mathrm{C}$ 、电场 30 40 kV/cm 条件下对样品极化 $15 \mathrm{~min}$, 测得压电 常数 $d_{33}$ 为 $300 \mathrm{pC} / \mathrm{N}$ 。经过阻抗分析测试发现, 中心 频率为 $2.24 \mathrm{MHz}$ 时，由固含量为质量分数 $81.8 \%$ 的 浆料制得的阵列具有 $-6 \mathrm{~dB}$ 的 $35 \%$ 带宽，这和传统 的 PZT 陶瓷传感器 $\left(-31 \%\right.$ 带宽) 类似。Tiller 等 ${ }^{[20]}$ 则 报道了光固化技术制备压电麦克风，其区别于前人 的亮点在于运用商业化的 3D 打印机完成包括导电 层、压电材料和高分子的复合器件制造。其中, 在 $X-Y$ 面的精度为 $27 \mu \mathrm{m}, Z$ 轴方向通过调整曝光参数也可 以将层厚精度控制在 $1 \mu \mathrm{m}$ 。虽然该工作制备的器件 性能 $\left(d_{33}\right.$ 约为 $\left.2 \sim 3 \mathrm{pC} / \mathrm{N}\right)$ 仅与溅射得到的氮化铝膜相 当，并且该工作中多种材料协同打印依靠人工停 止、更换原材料和机器再启动过程亦需要改进, 但 不可否认的是该工作具有革命性的意义，即作者以 麦克风为例，从原料制备、3D 建模、运用商业化的 增材制造设备制备不同几何形状的试样并对其进行 封装、测试和分析，完成了原材料一成型-器件的全流 程工作, 为今后增材制造压电器件指明了研究方向。

尽管光固化成型技术制备的样品表面光洁度高 且打印精度高, 但其需要的原材料用量大、成本高, 对于尺寸相对较小的压电陶瓷及器件而言, 还需对 增材制造设备进行针对性的设计, 以制备精度高且 尺寸小的压电陶瓷和器件。

\section{2 直写式打印}

作为一种较早发展起来的增材制造技术，直写 式打印(Direct ink writing, DIW)最先由美国桑迪亚 国家实验室于 1997 年提出 ${ }^{[46]}$, 并先后运用于陶瓷、 生物玻璃、细胞和生物组织等材料 ${ }^{[47-50]}$ 。在陶瓷增 材制造领域，直写式打印技术常见的原材料为剪切 变稀的黏弹性浆料。经过气动或电动压力作用, 浆 料通过微小内径的针头挤出后，以层叠的方式构建 三维模型。在已报道的文献中, 常用的针头内径范 围为 1 1000 $\mu \mathrm{m}^{[47-54]}$ 。与其他增材制造技术相比，该 技术可实现多材料打印，并且理论上对材料的种类 和数量没有限制 ${ }^{[22]}$ 。

直写式打印最初的应用与压电陶瓷相关。Tuttle 和 Smay 等 ${ }^{[55-56]}$ 首先将直写式打印应用于含铅压电
陶瓷。他们不仅详细阐述了制备过程，而且运用理 论分析不同连接方式(3-3、3-2、3-1)的样品单元对 压电性能的贡献。其中，直写式打印块体状 PZT 的 压电常数 $d_{33}$ 和 $d_{31}$ 分别为 604 和 $-269 \mathrm{pC} / \mathrm{N}$, 机电耦 合系数 $k_{31}$ 为 0.670 , 这与冷等静压制备的对照组样 品性能相近 $\left(\mathrm{d}_{33} \sim 573 \mathrm{pC} / \mathrm{N}, d_{31} \sim 255 \mathrm{pC} / \mathrm{N}, k_{31} \sim 0.645\right)$ 。 之后，他们使用不同尺寸针头将 PZT 打印制成线性 和环形, 测得的介电常数 $(1 \mathrm{kHz})$ 分别为 831 和 1081 , 压电常数 $d_{33}$ 分别为 388 和 $496 \mathrm{pC} / \mathrm{N}^{[57]}$ 。直写式打 印PZT 压电陶瓷的样品宏、微观形貌如图 4所示, 这 些样品用传统方法难以制备。

此后，研究人员探究了直写式打印无铅压电陶 瓷。 $\mathrm{Li}$ 等 ${ }^{[58]}$ 运用文献[43]的材料成分，即使用 $\mathrm{Li}$ 、 $\mathrm{Sb}$ 和 $\mathrm{Ta}$ 对 $\mathrm{KNN}$ 粉末进行掺杂，经过两步法煅烧的 技术路线制备原料粉末。该粉末在聚甲基丙烯酸甲 酯(Polymethyl methacrylate, PMMA)和季戊四醇三 丙烯酸酯中分散制备固含量为质量分数 $56 \%$ 的浆料, 进行直写式打印获得 3-3 连接方式的试样。经 $1100{ }^{\circ} \mathrm{C}$
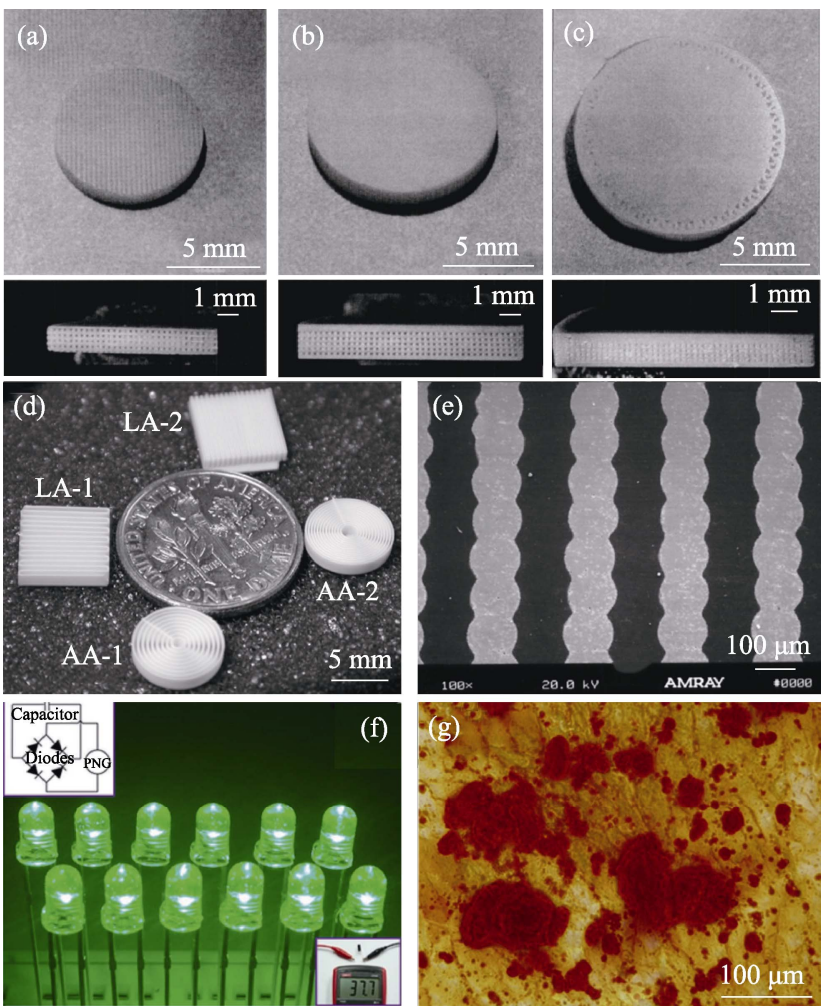

图 4 直写式打印压电陶瓷材料的宏观、微观形貌及应用

Fig. 4 Macrostructure, microstructure and application of piezoelectric ceramics prepared by direct ink writing (a-c) PZT in 3-3, 3-2 and 3-1 connectivity (upper and lower pictures show the surface and cross-section of the sample, respectively) ${ }^{[56]}$; (d) Linear and annular samples with a size bar of $5 \mathrm{~mm}$; (e) Cross-section of LA-2 in (d) ${ }^{[57]}$; (f) Captured image of 12 LEDs driven by the capacitor charged by KNN/PDMS ${ }^{[59]}$; (g) Alizarin red staining of $\mathrm{BST} / 40 \% \beta$-TCP composite, indicating the maximum mineral deposition with a good biomineralization activity ${ }^{[65]}$ 
烧结, 样品介电常数为 1775 。在 $2.5 \mathrm{kV} / \mathrm{mm}$ 电场下 对样品在 $100{ }^{\circ} \mathrm{C}$ 硅油中极化 $20 \mathrm{~min}$ 后, 压电常数 $d_{33}$ 为 $280 \mathrm{pC} / \mathrm{N}$, 剩余极化强度 $\left(P_{\mathrm{r}}\right)$ 为 $18.8 \mu \mathrm{C} / \mathrm{cm}^{2}$, 矫顽 场 $\left(E_{\mathrm{C}}\right)$ 为 $8.5 \mathrm{kV} / \mathrm{cm}$ 。 $\mathrm{GaO}$ 等 ${ }^{[59]}$ 将 $\mathrm{KNN}$ 纳米线分散 在聚二甲基硅氧烷和固化剂(质量比 10:1)组成的混 合物中, 制备固含量为质量分数 $40 \%$ 的浆料, 进而 制备压电纳米驱动器。该浆料打印在事先镀有 $\mathrm{Au} / \mathrm{Cr}$ 电极的聚对苯二甲酸乙二酯 (Polyethylene terephthalate, PET, $150 \mu \mathrm{m}$ )膜上, 在固化的压电陶 瓷层表面贴上一层镀有 $\mathrm{Au} / \mathrm{Cr}$ 的聚酰胺膜 $(50 \mu \mathrm{m})$, 两高分子膜之间的陶瓷层厚分别为一层、三层和五 层, 分别在 $0.4 、 1.2$ 和 $2.0 \mathrm{kV}$ 电压下进行极化。对 含有五层陶瓷的复合器件连接在电路中进行穿戴实 验和弯曲循环测试, 结果表明该器件收集到的能量 可以点亮 12 个串联的 LED, 如图 4(f)所示。

另一个重要的无铅压电体系 BT 也受到研究人 员的广泛关注。 Kim 等 ${ }^{[60]}$ 将 BT 粉末分散在聚偏氟 乙烯(Polyvinylidene fluoride, PVDF)和 $N, N$-二甲基 甲酰胺的混合物中制备浆料。在 $1 \mathrm{kHz}$ 条件下, 经 $1400{ }^{\circ} \mathrm{C}$ 烧结样品的介电常数为 4730 。在 $0.66 \mathrm{kV} / \mathrm{mm}$ 电场下对样品在 $80{ }^{\circ} \mathrm{C}$ 硅油中极化 $15 \mathrm{~h}$ 后, 测得压 电常数 $d_{33}$ 为 $200 \mathrm{pC} / \mathrm{N}$ 。Lorenz 等 ${ }^{[61]}$ 将中位径为 $1.2 \mu \mathrm{m}$ 的 BT 粉末分散在水中制备固含量为体积分 数 $50 \%$ 和 $52 \%$ 的浆料, 生坏干燥后借助冷等静压提 高样品的致密度。经过 $1350{ }^{\circ} \mathrm{C}$ 烧结 $3 \mathrm{~h}$ 后, 在 $1 \mathrm{kHz}$ 条件下测得样品的居里点为 $130{ }^{\circ} \mathrm{C}$ 。在 $2 \mathrm{kV} / \mathrm{mm}$ 电 场下对样品在硅油中极化 $30 \mathrm{~min}$ 后, 测得样品的常 温压电常数 $d_{33}$ 分别为 165 和 $200 \mathrm{pC} / \mathrm{N}$, 由此可见, 浆料固含量和生坏致密度的差异会影响样品的最 终致密度, 直接体现为压电常数的差距。

近年来, $\mathrm{BT}$ 经 $\mathrm{Ca}$ 和 $\mathrm{Zr}$ 共掺杂的材料体系 $\mathrm{Ba}_{0.85} \mathrm{Ca}_{0.15} \mathrm{Zr}_{0.1} \mathrm{Ti}_{0.9} \mathrm{O}_{3}(\mathrm{BCZT})$ 因较高的压电常数 $\left(d_{33} \sim 620 \mathrm{pC} / \mathrm{N}\right)$ 得到了广泛关注 ${ }^{[62]}$ 。研究人员运用直 写式打印制备了 3-3 和 1-3 连接方式的 $\mathrm{BCZT}^{[63-64]}$, 通过调整粉末粒度分布和分散剂添加量, 提高悬浊 液的稳定性, 并调整黏结剂和絮凝剂的添加量, 得 到适合 $150 \sim 410 \mu \mathrm{m}$ 针头挤出的浆料。经 $1500{ }^{\circ} \mathrm{C}$ 烧 结的 3-3 试样在 $1 \mathrm{kHz}$ 条件下介电常数为 1046 , 常 温介电损耗为 0.021 , 压电常数 $d_{33}$ 为 $(100 \pm 4) \mathrm{pC} / \mathrm{N}$ 。 同时, 研究人员对比喷墨打印过程中的按需送料 (Drop-on-demand, DOD) 原理, 首次提出按需送丝 (Filament-on-demand, FOD) 的打印模式, 实现阵列 状 1-3 试样的成功制备。

类似地, $\mathrm{Sr}$ 掺杂 $\mathrm{BT}$ 的体系 $(\mathrm{Ba}, \mathrm{Sr}) \mathrm{TiO}_{3}(\mathrm{BST})$ 和 $\beta$-磷酸三钙 $(\beta-\mathrm{TCP})$ 的复合多孔支架也由直写式打 印法成功制备 ${ }^{[65]}$, 以期用于下一代骨组织工程中。
$\mathrm{BST} /(x) \beta-\mathrm{TCP}(x=0,10 \%, 20 \%, 30 \%, 40 \%, 50 \%$, 均 为质量分数)粉末与 $\mathrm{PVC}$ 溶液混合制备成浆料, 通 过直写式技术打印为多孔试样, 其中 $\beta$-TCP 含量为 $40 \%$ 的组分具有最高的密度和力学性能指标, 随着 $\beta$-TCP 添加量提高, 样品介电性能下降。同时, 该成 分的多孔试样在模拟体液中浸泡 $28 \mathrm{~d}$ 后显示良好的 生物活性和相容性, 如图 4(g)所示, 对样品表面磷 灰石矿化程度分析可知, 随着 $\beta$-TCP 含量增大, 样 品表面的羟基磷灰石含量随之增大。由此可见, $\mathrm{BST} / 40 \% \beta-\mathrm{TCP}$ 试样兼具良好的生物相容性和力学 性能, 适合成骨细胞生长。运用增材技术制造无铅 压电陶瓷和生物材料构成的复合材料, 为今后新型 超声辅助成骨细胞生长的研究提供了新的思路。

与光固化技术相比, 虽然直写式打印的样品表 面粗龯度较高且打印过程会出现堵头现象导致打印 中断，但是其多材料同时制备的优势更适合压电器 件的制备。故直写式打印也将成为今后多材料增 材制造的重要发展方向。

\section{3 喷墨打印}

喷墨打印是现代印刷中运用最广的技术。1951 年, 第一个成功使用喷射墨水的产品由 Elmquist 在瑞典 发明, 随后, 研究人员分别于 1967 年和 1973 年发明 了喷墨打印机和两种电信号控制的喷墨技术 ${ }^{[24]}$ 。喷墨 打印机主要分为连续喷射(Continuous-jet)和按需喷 射(Drop-on-demand, DOD)两种模式, 为了形成气压 脉冲实现液滴的按需喷射, 在 DOD 模式下, 喷头又 可以分为加热喷射和压电喷射两种机制 ${ }^{[66]}$ 。喷墨打 印陶瓷的报道始于 20 世纪 90 年代, 主要以 $\mathrm{ZrO}_{2}$ 、 $\mathrm{TiO}_{2}$ 等陶瓷为主 ${ }^{[67-70]}$ 。

喷墨打印压电陶瓷的连接方式主要为 2-2 和 1-3。Bhatti 等 ${ }^{[71]}$ 通过混合乙醇、异丙醇、表面活性 剂、黏结剂作为分散介质制备 PZT 浆料, 喷墨打印 1-3 连接方式的 PZT 柱状阵列, 阐述了浆料的配制 方法和与之匹配的打印参数, 并尝试增加打印层数 以提高阵列单元的高径比(如图 5 所示)。之后, 法国 研究人员深入讨论了 1-3 连接方式的 PZT 喷墨打印 工艺 ${ }^{[72-74]}$, 对浆料提出三点要求: 1)减少沉降; 2)控 制浆料干燥过程，降低液滴喷射频率同时防止堵孔; 3)调整流体性能, 包括粘度和表面张力。在后续的 工作中, 他们还结合打印工艺(液滴打印速度、液滴 体积、喷嘴尺寸等), 利用激光干涉仪对单个液滴成 型的柱状单元进行断层摄影分析, 寻找适合的浆料 粘度范围以及与之匹配的打印参数。

近年来, 斯洛文尼亚的研究人员摒弃有机溶剂 

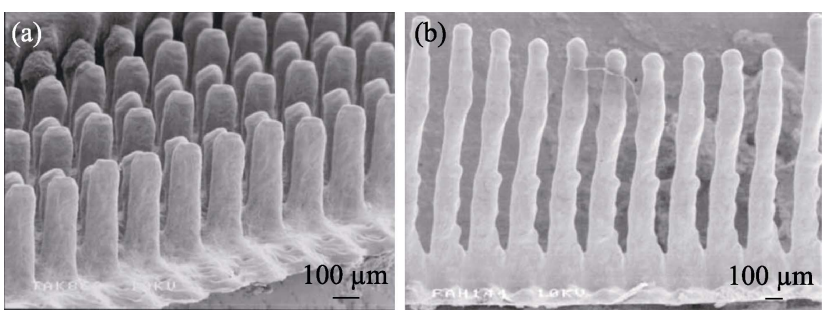

图 5 喷墨打印柱状阵列高度差异 ${ }^{[71]}$

Fig. 5 Different heights of pillar arrays made by ink-jet printing ${ }^{[71]}$ (a) Sample printed in 1000 layers; (b) Sample printed in 4000 layers

而使用水作为浆料的分散介质，日本的研究人员则 直接使用陶瓷前驱体作为原料进行喷墨打印。研究 人员 ${ }^{[75-77]}$ 对自制原料粉末和浆料进行分析, 从粉末 粒径分布、红外分析和热分析结果入手调整粉末研 磨时间, 测试墨水的 Zeta 电位、粘度, 优化墨水成 分及其稳定性。喷墨打印的膜经 $850{ }^{\circ} \mathrm{C}$ 烧结 $2 \mathrm{~h}$ 后, 膜厚为 $15 \mu \mathrm{m}, 1 \mathrm{kHz}$ 频率下测得的介电常数为 190 , 在 $4 \mathrm{kV} / \mathrm{mm}$ 电场、 $120{ }^{\circ} \mathrm{C}$ 硅油中对样品极化 $40 \mathrm{~min}$ 后 测得的压电常数 $e_{33}=3.5 \mathrm{C} / \mathrm{m}^{2}$, 机电耦合因数 $k_{\mathrm{t}}=0.46$ 。 Wagata 等 ${ }^{[78]}$ 使用 BT 前驱体溶液进行喷墨打印, 从 根本上避免堵孔的发生。陶瓷前驱体通过 $60 \mu \mathrm{m}$ 直 径的喷嘴直接沉积于 $360{ }^{\circ} \mathrm{C}$ 的热玻璃上形成圆点。 如果玻璃温度低于 $360{ }^{\circ} \mathrm{C}$, 液滴沉积后发生分散, 圆点的直径偏差大。此外, 研究人员还通过控制液 滴浓度和体积制备不同直径的点阵。陶瓷前驱体随 着玻璃温度提高, $\mathrm{X}$ 射线衍射谱图逐渐由非晶相变 为晶相, 到 $650{ }^{\circ} \mathrm{C}$ 成为完全的 $\mathrm{BT}$ 衍射峰。

喷墨打印与直写式打印都是采用液体浆料的直 接打印, 两者的最明显差异在于浆料粘度: 喷墨打印 浆料的粘度一般小于 $<20 \mathrm{mPa} \cdot \mathrm{s}$, 而直写式打印的浆 料粘度一般大于 $1000 \mathrm{~Pa} \cdot \mathrm{s}$ (剪切率为 $\left.0.1 \mathrm{~s}^{-1}\right)^{[25,64,79-83]}$ 。 喷墨打印虽起步早, 但发展较慢, 大多数工作只停 留在陶瓷成型层面, 性能报道较少。近年来, 直写式 打印从材料和成型角度的发展较快, 相关的功能性 能报道较多。为避免喷嘴堵孔并提高成型精度, 陶 瓷前驱体原料将成为直写式打印和喷墨打印的研究 方向。

\section{4 熔融沉积成型}

熔融沉积成型是目前商业化程度最高的增材制 造技术，目前市面上常见的原材料以丙烯腈-丁二 烯一苯乙烯共聚物 (Acrylonitrile butadiene styrene, ABS)、聚乳酸(Polylactic acid, PLA)和尼龙等高分子 聚合物丝材为主，其他材料亦可通过小批量订制 获得。
美国罗格斯大学的 Safari 团队 ${ }^{[27,82-89]}$ 最早将该 技术运用于含铅压电陶瓷成型领域。他们通过调整 复合丝材的材料组成寻找适于打印的成分配比, 结 果表明, PZT 丝材的固含量为体积分数 $55 \%$, 通过 逐层沉积可以直接制备线形试样(Linear)和辐射状 (Radial)的压电致动器。FDM 的生坏含有大量有机 物，因此如何顺利脱除有机物、保证试样高致密度 是该技术在后处理过程中的难点 ${ }^{[84]}$ 。同时，研究人 员还将该 PZT 丝材打印成 2-2 连接方式的环形试样 和 3-3 连接方式的多孔试样, 并借助 FDM 打印 1-3 连接方式的模具, 运用粉浆浇筑技术制备 1-3 连接 方式的 PZT 试样, 如图 6 所示。经 $1285^{\circ} \mathrm{C}$ 烧结, 3-3 试样和 2-2 试样的介电常数分别为 700 和 $627^{[85]}$ 。 经电晕极化后, 3-3、1-3、2-2 试样的压电常数 $d_{33}$ 分别为 $(290 \pm 10) 、(280 \pm 10)^{[85]}$ 和(397 \pm 16$) \mathrm{pC} / \mathrm{N}^{[86]}$ 。

在无铅体系方面，现阶段报道多以 BT 体系为 主。Castles 等 ${ }^{[28]}$ 通过制备不同固含量的 $\mathrm{BT} / \mathrm{ABS}$ 复
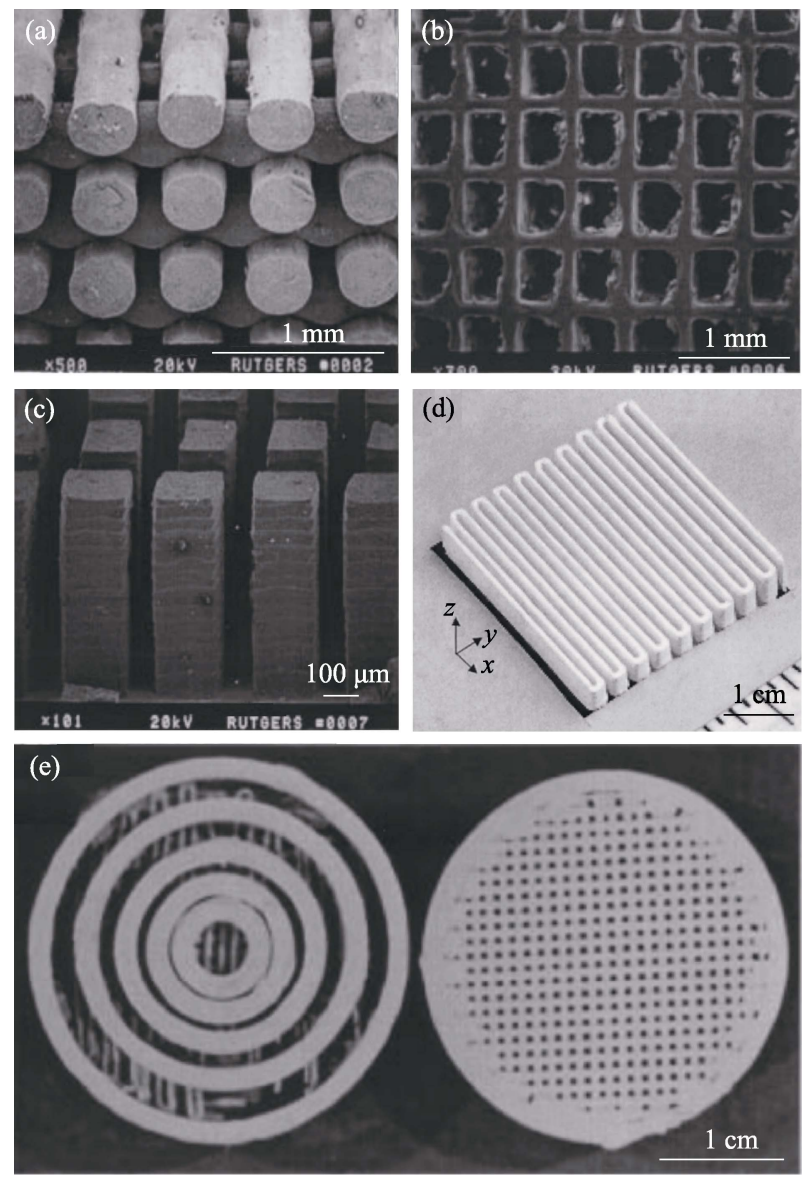

图 6 熔融沉积成型的部分样品的宏观、微观形貌

Fig. 6 Macrostructures and microstructures of samples prepared by fused deposition modelling

(a) 3-3 porous ladder sample ${ }^{[85]}$; (b) Wax mould ${ }^{[85]}$; (c) 1-3 pillar arrays made by lost mould process (mould in (b) $)^{[85]}$; (d) 2-2 linear sample ${ }^{[86]}$; (e) Left showing 2-2 annular ring and right showing 3-3 ladder structures $^{[85]}$ 
合丝材(固含量为质量分数 $0 \sim 70 \%$ ) 打印不同连接方 式的 BT, 并分析制备过程中产生缺陷的原因。所有 试样均未经烧结, 介电常数低 $(<10)$, 介电损耗大 $(>0.004)$ 。 Kim 等 ${ }^{[90]}$ 将 $\mathrm{BT}$ 与 PVDF 进行复合, 制备 丝材, 再用 FDM 制备厚度为 $0.33 \mathrm{~mm}$ 的厚膜, 用直 接混合 BT/PVDF 成为浆料通过流延成型制备的试 样作为参照组试样。两种方法制备的厚膜均在 $90{ }^{\circ} \mathrm{C}$ 硅油中极化 $2 \mathrm{~h}$, 极化电场为 $35 \mathrm{kV} / \mathrm{mm}$ 。其中, FDM 试样的压电常数 $d_{31}$ 为 $2.1 \times 10^{-2} \mathrm{pC} / \mathrm{N}$, 而流延 成型试样的 $d_{31}$ 仅为 $9.0 \times 10^{-3} \mathrm{pC} / \mathrm{N}$ 。本文中部分参 考文献报道的增材制造压电陶瓷的功能性能总结 于表 2。

虽然 FDM 是目前最容易获得的增材制造设备, 但是市面上的压电陶瓷丝材不多见。由于复合丝材 含有大量高分子材料, 如何保证打印后样品的功能 性能接近压电陶瓷的本征性能是目前亟需解决的问 题。同时, 由于熔融沉积成型的大部分设备喷嘴精 度不高, 导致打印试样的几何精度不高, 这也是该 技术今后亟待攻克的方向。

\section{5 总结与展望}

本文探讨了目前压电陶瓷的增材制造技术，从 各种技术的发展历史、已报道的材料体系入手, 对 增材制造压电陶瓷原材料的制造工艺、样品的性能 表征及其应用进行阐述、对比。从材料体系而言, 增 材制造压电陶瓷从含铅体系逐步发展到以 $\mathrm{BT}$ 和 $\mathrm{KNN}$ 体系为主、其他掺杂体系为辅的无铅体系。从
连接方式而言, 样品以 3-3、1-3、2-2 为主, 引入超 材料结构可以带来新的思路。光固化和直写式打印 比喷墨打印和熔融沉积成型的学术型研究更多, 这 从侧面反映了增材制造的方向一一即高精度和多材 料制造。从功能性能而言, 部分样品的介电、压电 性能已达到与本征性能相近的水平, 但是增材制造 样品的综合性能仍有提升空间。虽然现阶段黏结剂 喷射技术应用于压电陶瓷领域的文献较少, 本文未 予评述，但不可否认的是，在工业界通用、惠普、武 汉易制等国内外企业都已推出针对黏结剂喷射技术 的 3D 打印陶瓷解决方案, 发展速度迅猛。

尽管增材制造压电陶瓷的成型问题已基本解决, 下一步该领域的研究仍需要从细节入手, 在原料优 化、几何外形的合理设计、样品后处理等方面努力, 以改善增材制造试样的功能性能, 为增材制造压电 器件打好基础。原材料的制备过程需要提高纳米粉 体的分散性和浆料的稳定性, 使用陶瓷前驱体作为 原料实现更高精度样品的制备, 同时多材料打印也 有利于推动有机一无机压电复合材料的发展。几何外 形设计要在现有 $3 \mathrm{D}$ 设计切片软件的基础上, 将增 材制造工艺与软件编程相结合, 实现路径的最优化 设计。在样品后处理方面, 从固化工艺或排胶、烧 结工艺入手, 避免不合理工艺带来的缺陷, 保证样 品的致密度。性能测试前的极化工艺包括极化方法、 极化电压、极化时间等需要大量实验验证, 以发挥 试样的综合性能。另外, 由于压电陶瓷在外场作用 下产生微小的尺寸变化, 故在电场作用下的 4D 打 印压电陶瓷也是值得探索的方向。

表 2 增材制造压电陶瓷功能性能

Table 2 Functional properties of piezoelectric ceramics made by AM

\begin{tabular}{|c|c|c|c|c|c|c|c|c|}
\hline Material & AM techniques & Connectivities & $\varepsilon_{\mathrm{r}}$ & $\tan \delta$ & Poling conditions & $d_{33} /\left(\mathrm{pC} \cdot \mathrm{N}^{-1}\right)$ & $k_{\mathrm{t}}$ & Ref. \\
\hline BT & VP & $1-3$ & 1350 & - & $30 \mathrm{kV} \cdot \mathrm{cm}^{-1}, 100{ }^{\circ} \mathrm{C} 30 \mathrm{~min}$ & 160 & 0.474 & {$[40]$} \\
\hline BT & VP & $1-3$ & 920 & 0.07 & $2 \mathrm{~V} \cdot \mu \mathrm{m}^{-1}, 120{ }^{\circ} \mathrm{C} 30 \mathrm{~min}$ & 87 & 0.3 & [19] \\
\hline PZT & VP & $1-3$ & 1040 & 0.020 & $\begin{array}{l}30-40 \mathrm{kV} \cdot \mathrm{cm}^{-1}, 70{ }^{\circ} \mathrm{C} 15 \mathrm{~min} \text {, } \\
\text { silicone oil }\end{array}$ & 345 & 0.53 & [45] \\
\hline PZT & DIW & Concentric ring & 1081 & - & $\begin{array}{l}25 \mathrm{kV} \cdot \mathrm{cm}^{-1} \text {, room temperature } \\
30 \mathrm{~min}\end{array}$ & 496 & - & [57] \\
\hline KNN & DIW & $3-3$ & 1775 & - & $\begin{array}{l}2.5 \mathrm{kV} \cdot \mathrm{mm}^{-1}, 100{ }^{\circ} \mathrm{C} 20 \mathrm{~min} \text {, } \\
\text { silicone oil }\end{array}$ & 280 & - & {$[58]$} \\
\hline BT & DIW & Bulk & 4730 & 0.033 & $\begin{array}{l}0.66 \mathrm{MV} \cdot \mathrm{m}^{-1}, 80{ }^{\circ} \mathrm{C} \quad 15 \mathrm{~h}, \\
\text { silicone oil }\end{array}$ & 200 & - & {$[60]$} \\
\hline BCZT & DIW & $3-3$ & 1046 & 0.021 & $\begin{array}{l}3 \mathrm{kV} \cdot \mathrm{mm}^{-1} \text {, room temperature } \\
30 \mathrm{~min} \text {, silicone oil }\end{array}$ & $(100 \pm 4)$ & - & {$[63]$} \\
\hline $\mathrm{Nb}-\mathrm{PZT}$ & IP & $2-2$ & $\sim 700$ & $\sim 0.04$ & $\begin{array}{l}4 \mathrm{kV} \cdot \mathrm{mm}^{-1}, 120{ }^{\circ} \mathrm{C} \quad 40 \mathrm{~min} \text {, } \\
\text { silicone oil }\end{array}$ & - & 0.46 & {$[77]$} \\
\hline PZT & FDM & $3-3$ & 700 & - & $\begin{array}{l}25 \mathrm{kV}, 70{ }^{\circ} \mathrm{C} 15-20 \mathrm{~min} \text {, Cor- } \\
\text { ona technique }\end{array}$ & $(290 \pm 10)$ & 0.5 & [85] \\
\hline PZT & FDM & $2-2$ & 627 & 0.023 & $\begin{array}{l}26 \mathrm{kV}, 60{ }^{\circ} \mathrm{C} 15 \mathrm{~min} \text {, Corona } \\
\text { technique }\end{array}$ & $(397 \pm 16)$ & $0.68,0.32\left(k_{\mathrm{p}}\right)$ & [86] \\
\hline
\end{tabular}




\section{参考文献:}

[1] XU S, HANSEN B J, WANG Z L. Piezoelectric-nanowire-enabled power source for driving wireless microelectronics. Nature Communications, 2010, 1: 93.

[2] VAN DEN ENDE D A, VAN DE WIEL H J, GROEN W A, et al. Direct strain energy harvesting in automobile tires using piezoelectric PZT-polymer composites. Smart Materials and Structures, 2012, 21: 015011.

[3] XU X H, LI H. Photoacoustic imaging in biomedicine. Physics, 2008, 37(2): 111-119.

[4] CARULlO A, PARVIS M. An ultrasonic sensor for distance measurement in automotive applications. IEEE Sensors Journal, 2001, 1(2): 143.

[5] ROUNDY S, WRIGHT P K. A piezoelectric vibration based generator for wireless electronics. Smart Materials and Structures, 2004, 13(5): 1131.

[6] SWARTZ S L. Topics in electronic ceramics. IEEE Transactions on Electrical Insulation, 1990, 25(5): 935-987.

[7] MATTOX J M. Additive manufacturing and its implications for military ethics. Journal of Military Ethics, 2013, 12(3): 225-234.

[8] IBN-MOHAMMED T, KOH S C L, REANEY I M, et al. Integrated hybrid life cycle assessment and supply chain environmental profile evaluations of lead-based (lead zirconate titanate) versus lead-free (potassium sodium niobate) piezoelectric ceramics. Energy \& Environmental Science, 2016, 9(11): 3495-3520.

[9] BELL A J, DEUBZER O. Lead-free piezoelectrics-the environmental and regulatory issues. MRS Bulletin, 2018, 43(8): 581-587.

[10] NEWNHAM R E, SKINNER D P, CROSS L E. Connectivity and piezoelectric-pyroelectric composites. Materials Research Bulletin, 1978, 13(5): 525-536.

[11] JANTUNEN H, HU T, UUSIMÄKI A, et al. Tape casting of ferroelectric, dielectric, piezoelectric and ferromagnetic materials. Journal of the European Ceramic Society, 2004, 24(6): 1077-1081.

[12] PARK G T, CHOI J J, PARK C S, et al. Piezoelectric and ferroelectric properties of $1-\mu \mathrm{m}$-thick lead zirconate titanate film fabricated by a double-spin-coating process. Applied Physics Letters, 2004, 85(12): 2322-2324.

[13] SAVAKUS H P, KLICKER K A, NEWNHAM R E. PZT-epoxy piezoelectric transducers: a simplified fabrication procedure. Materials Research Bulletin, 1981, 16(6): 677-680.

[14] GARCÍA-GANCEDO L, OLHERO S M, ALVES F J, et al. Application of gel-casting to the fabrication of 1-3 piezoelectric ceramic-polymer composites for high-frequency ultrasound devices. Journal of Micromechanics and Microengineering, 2012, 22(12): 125001.

[15] LEJEUNE M, CHARTIER T, DOSSOU-YOVO C, et al. Ink-jet printing of ceramic micro-pillar arrays. Advances in Science and Technology, 2006, 45: 413-420.

[16] ICHIDA Y. Current status of 3D printer use among automotive suppliers: can 3D printed-parts replace cast parts. IFEAMA SPSCP, 2016, 5: 69-82.

[17] NICHOLS M R. How does the automotive industry benefit from 3D metal printing? Metal Powder Report, 2019, 74(5): 257-258.

[18] LAMBERT P, CHARTRAIN N, SCHULTZ A, et al. Mask Projection Microstereolithography of Novel Biocompatible Polymers. International Solid Freeform Fabrication Symposium, 2014: 974-990.

[19] SONG X, CHEN Z, LEI L, et al. Piezoelectric component fabrication using projection-based stereolithography of barium titanate ceramic suspensions. Rapid Prototyping Journal, 2017, 23(1): 44-53.

[20] TILLER B, REID A, ZHU B, et al. Piezoelectric microphone via a digital light processing 3D printing process. Materials \& Design, 2019, 165: 107593.

[21] OVHAL M M, KUMAR N, KANG J W. 3D direct ink writing fabrication of high-performance all-solid-state micro-supercapacitors. Molecular Crystals and Liquid Crystals, 2020, 705(1): 105-111.

[22] TRUBY R L, LEWIS J A. Printing soft matter in three dimensions. Nature, 2016, 540(7633): 371-378.

[23] CHU Y, QIAN C, CHAHAL P, et al. Printed diodes: materials processing, fabrication, and applications. Advanced Science, 2019, 6(6): 1801653.

[24] HEINZL J, HERTZ C H. Ink-jet printing. Advances in Electronics and Electron Physics, 1985, 65: 91-171.

[25] WANG T, DERBY B. Ink-jet printing and sintering of PZT. Journal of the American Ceramic Society, 2005, 88(8): 2053-2058.

[26] LEE K Y, CHO J W, CHANG N Y, et al. Accuracy of three-dimensional printing for manufacturing replica teeth. The Korean Journal of Orthodontics, 2015, 45(5): 217-225.

[27] SAFARI A, CESARANO J, CLEM P G, et al. Fabrication of Advanced Functional Electroceramic Components by Layered Manufacturing (LM) Methods. Proceedings of the 13th IEEE International Symposium on Applications of Ferroelectrics, 2002. ISAF, Japan, 2002: 1-6.

[28] CASTLES F, ISAKOV D, LUI A, et al. Microwave dielectric characterisation of 3D-printed $\mathrm{BaTiO}_{3} / \mathrm{ABS}$ polymer composites. Scientific Reports, 2016, 6: 22714.

[29] PARUPELLI S, DESAI S. A comprehensive review of additive manufacturing (3D printing): processes, applications and future potential. American Journal of Applied Sciences, 2019, 16(8): 244-272.

[30] BÁRTOLO P J. Stereolithography: Materials, Processes and Applications. Boston: Springer Science \& Business Media, 2011: 42.

[31] SWAINSON W K. Method, Medium and Apparatus for Producing Three-dimensional Figure Product. US Patent, 4041476. 1977-08-09.

[32] HULL C W. Apparatus for Production of Three-dimensional Objects by Stereolithography. US Patent, 45753301. 1986-03-11.

[33] HULL C W, SPENCE S T, ALBERT D J, et al. Methods and Apparatus for Production of Three-dimensional Objects by Stereolithography. US Patent, 5059359. 1991-10-22.

[34] LI S, DUAN W, ZHAO T, et al. The fabrication of SiBCN ceramic components from preceramic polymers by digital light processing (DLP) 3D printing technology. Journal of the European Ceramic Society, 2018, 38(14): 4597-4603.

[35] HE C, LIU X, MA C, et al. Digital light processing fabrication of mullite component derived from preceramic precursor using photosensitive hydroxysiloxane as the matrix and alumina nanoparticles as the filler. Journal of the European Ceramic Society, 2021, 41(11): 5570-5577

[36] DUFAUD O, MARCHAL P, CORBEL S. Rheological properties of PZT suspensions for stereolithography. Journal of the European Ceramic Society, 2002, 22(13): 2081-2092.

[37] DUFAUD O, CORBEL S. Oxygen diffusion in ceramic suspensions for stereolithography. Chemical Engineering Journal, 2003, 92(1/2/3): 55-62.

[38] DUFAUD O, CORBEL S. Application of stereolithography to chemical engineering: 'from macro to micro'. Chemical Engineering Research and Design, 2005, 83(2): 133-138.

[39] KIM K, ZHU W, QU X, et al. 3D optical printing of piezoelectric nanoparticle-polymer composite materials. ACS Nano, 2014, 8(10): 
9799-9806.

[40] CHEN Z, SONG X, LEI L, et al. 3D printing of piezoelectric element for energy focusing and ultrasonic sensing. Nano Energy, 2016, 27: 78-86.

[41] VIJATOVIĆ M M, BOBIĆ J D, STOJANOVIĆ B D. History and challenges of barium titanate: Part II. Science of Sintering, 2008, 40(3): 235-244.

[42] CHEN W, WANG F, YAN K, et al. Micro-stereolithography of KNN-based lead-free piezoceramics. Ceramics International, 2019 , 45(4): 4880-4885.

[43] SAITO Y, TAKAO H, TANI T, et al. Lead-free piezoceramics. Nature, 2004, 432(7013): 84-87.

[44] CUI H, HENSLEIGH R, YAO D, et al. Three-dimensional printing of piezoelectric materials with designed anisotropy and directional response. Nature Materials, 2019, 18(3): 234-241.

[45] CHEN Y, BAO X, WONG C M, et al. PZT ceramics fabricated based on stereolithography for an ultrasound transducer array application. Ceramics International, 2018, 44(18): 22725-22730.

[46] CESARANO III J, CALVERT P D. Freeforming Objects with Low-binder Slurry. US Patent, 6027326. 2000-02-22.

[47] MORISSETTE S L, LEWIS J A, CESARANO J, et al. Solid freeform fabrication of aqueous alumina-poly(vinyl alcohol) gelcasting suspensions. Journal of the American Ceramic Society, 2000, 83(10): 2409-2416.

[48] DUOSS E B, TWARDOWSKI M, LEWIS J A. Sol-Gel inks for direct-write assembly of functional oxides. Advanced Materials, 2007, 19(21): 3485-3489.

[49] MARQUES C F, PERERA F H, MAROTE A, et al. Biphasic calcium phosphate scaffolds fabricated by direct write assembly: mechanical, anti-microbial and osteoblastic properties. Journal of the European Ceramic Society, 2017, 37(1): 359-368.

[50] KOLESKY D B, TRUBY R L, GLADMAN A S, et al. 3D bioprinting of vascularized, heterogeneous cell-laden tissue constructs. Advanced Materials, 2014, 26(19): 3124-3130.

[51] KOLESKY D B, HOMAN K A, SKYLAR-SCOTT M A, et al. Three-dimensional bioprinting of thick vascularized tissues. Proceedings of the National Academy of Sciences, 2016, 113(12): 3179-3184.

[52] SMAY J E, CESARANO J, LEWIS J A. Colloidal inks for directed assembly of 3-D periodic structures. Langmuir, 2002, 18(14): 5429-5437.

[53] WANG R, ZHU P, YANG W, et al. Direct-writing of 3D periodic $\mathrm{TiO}_{2}$ bio-ceramic scaffolds with a Sol-Gel ink for in vitro cell growth. Materials \& Design, 2018, 144: 304-309.

[54] CHEN T, SUN A, CHU C, et al. Rheological behavior of titania ink and mechanical properties of titania ceramic structures by 3D direct ink writing using high solid loading titania ceramic ink. Journal of Alloys and Compounds, 2019, 783: 321-328.

[55] TUTTLE B A, SMAY J E, CESARANO J, et al. Robocast $\mathrm{Pb}\left(\mathrm{Zr}_{0.95} \mathrm{Ti}_{0.05}\right) \mathrm{O}_{3}$ ceramic monoliths and composites. Journal of the American Ceramic Society, 2001, 84(4): 872-874.

[56] SMAY J E, CESARANO III J, TUTTLE B A, et al. Piezoelectric properties of 3-X periodic $\mathrm{Pb}\left(\mathrm{Zr}_{x} \mathrm{Ti}_{1-x}\right) \mathrm{O}_{3}$-polymer composites. Journal of Applied Physics, 2002, 92(10): 6119-6127.

[57] SMAY J E, CESARANO III J, TUTTLE B A, et al. Directed colloidal assembly of linear and annular lead zirconate titanate arrays. Journal of the American Ceramic Society, 2004, 87(2): 293-295.

[58] LI Y, LI L, LI B. Direct ink writing of three-dimensional (K, Na) $\mathrm{NbO}_{3}-$ based piezoelectric ceramics. Materials, 2015, 8(4): 1729-1737.

[59] GAO M, LI L, LI W, et al. Direct writing of patterned, lead-free nanowire aligned flexible piezoelectric device. Advanced Science,
2016, 3(8): 1600120

[60] KIM H, RENTERIA-MARQUEZ A, ISLAM M D, et al. Fabrication of bulk piezoelectric and dielectric $\mathrm{BaTiO}_{3}$ ceramics using paste extrusion 3D printing technique. Journal of the American Ceramic Society, 2019, 102(6): 3685-3694.

[61] LORENZ M, MARTIN A, WEBBER K G, et al. Electromechanical properties of Robocasted barium titanate ceramics. Advanced Engineering Materials, 2020, 22(9): 2000325.

[62] LIU W, REN X. Large piezoelectric effect in Pb-free ceramics. Physical Review Letters, 2009, 103(25): 257602.

[63] NAN B, OLHERO S, PINHO R, et al. Direct ink writing of macroporous lead-free piezoelectric $\mathrm{Ba}_{0.85} \mathrm{Ca}_{0.15} \mathrm{Zr}_{0.1} \mathrm{Ti}_{0.9} \mathrm{O}_{3}$. Journal of the American Ceramic Society, 2019, 102(6): 3191-3203.

[64] NAN B, GALINDO-ROSALES F J, FERREIRA J M F. 3D printing vertically: direct ink writing free-standing pillar arrays. Materials Today, 2020, 35: 16-24.

[65] TARIVERDIAN T, BEHNAMGHADER A, MILAN P B, et al. 3D-printed barium strontium titanate-based piezoelectric scaffolds for bone tissue engineering. Ceramics International, 2019, 45(11): 14029-14038.

[66] DERBY B. Inkjet printing of functional and structural materials: fluid property requirements, feature stability, and resolution. Annual Review of Materials Research, 2010, 40: 395-414.

[67] BLAZDELL P F, EVANS J R G, EDIRISINGHE M J, et al. The computer aided manufacture of ceramics using multilayer jet printing. Journal of Materials Science Letters, 1995, 14(22): $1562-1565$.

[68] XIANG Q F, EVANS J R G, EDIRISINGHE M J, et al. Solid freeforming of ceramics using a drop-on-demand jet printer. Proceedings of the Institution of Mechanical Engineers, Part B: Journal of Engineering Manufacture, 1997, 211(3): 211-214.

[69] ZHAO X, EVANS J R G, EDIRISINGHE M J, et al. Direct ink-jet printing of vertical walls. Journal of the American Ceramic Society, 2002, 85(8): 2113-2115.

[70] TENG W D, EDIRISINGHE M J, EVANS J R G. Optimization of dispersion and viscosity of a ceramic jet printing ink. Journal of the American Ceramic Society, 1997, 80(2): 486-494.

[71] BHATTI A R, MOTT M, EVANS J R G, et al. PZT pillars for 1-3 composites prepared by ink-jet printing. Journal of Materials Science Letters, 2001, 20(13): 1245-1248.

[72] NOGUERA R, DOSSOU-YOVO C, LEJEUNE $\mathrm{M}$, et al. Fabrication of 3D fine scale PZT components by ink-jet prototyping process. Journal de Physique IV (Proceedings), 2005, 128: 87-93.

[73] NOGUERA R, LEJEUNE M, CHARTIER T. 3D fine scale ceramic components formed by ink-jet prototyping process. Journal of the European Ceramic Society, 2005, 25(12): 2055-2059.

[74] LEJEUNE M, CHARTIER T, DOSSOU-YOVO C, et al. Ink-jet printing of ceramic micro-pillar arrays. Journal of the European Ceramic Society, 2009, 29(5): 905-911.

[75] NOSHCHENKO O, KUSCER D, MOCIOIU O C, et al. Effect of milling time and $\mathrm{pH}$ on the dispersibility of lead zirconate titanate in aqueous media for inkjet printing. Journal of the European Ceramic Society, 2014, 34(2): 297-305.

[76] BAKARIČ T, MALIČ B, KUSCER D. Lead-zirconate-titanatebased thick-film structures prepared by piezoelectric inkjet printing of aqueous suspensions. Journal of the European Ceramic Society, 2016, 36(16): 4031-4037.

[77] KUSCER D, DRNOVŠEK S, LEVASSORT F. Inkjet-printingderived lead-zirconate-titanate-based thick films for printed electronics. Materials \& Design, 2020, 198: 109324.

[78] WAGATA H, GALLAGE R, YOSHIMURA M, et al. Patterning of 
$\mathrm{BaTiO}_{3}$ by inkjet deposition using a precursor solution. Materials Science and Engineering: B, 2009, 161(1/2/3): 146-150.

[79] SEERDEN K A, REIS N, EVANS J R, et al. Ink-jet printing of wax-based alumina suspensions. Journal of the American Ceramic Society, 2001, 84(11): 2514-2520.

[80] VADILlO D C, TUladHAR T R, MULJI A C, et al. The rheological characterization of linear viscoelasticity for ink jet fluids using piezo axial vibrator and torsion resonator rheometers. Journal of Rheology, 2010, 54(4): 781-795.

[81] LEWIS J A. Direct ink writing of 3D functional materials. Advanced Functional Materials, 2006, 16(17): 2193-2204.

[82] SCHLORDT T, SCHWANKE S, KEPPNER F, et al. Robocasting of alumina hollow filament lattice structures. Journal of the European Ceramic Society, 2013, 33: 3243-3248.

[83] MUTH J T, VOGT D M, TRUBY R L, et al. Embedded 3D printing of strain sensors within highly stretchable elastomers. Advanced Materials, 2014, 26(36): 6307-6312.

[84] MCNULTY T F, MOHAMMADI F, BANDYOPADHYAY A, et al. Development of a binder formulation for fused deposition of ceramics. Rapid Prototyping Journal, 1998, 4(4): 144-150.

[85] BANDYOPADHYAY A, PANDA R K, MCNULTY T F, et al. Piezoelectric ceramics and composites via rapid prototyping techniques. Rapid Prototyping Journal, 1998, 4(1): 37-49.

[86] LOUS G M, CORNEJO I A, MCNULTY T F, et al. Fabrication of piezoelectric ceramic/polymer composite transducers using fused deposition of ceramics. Journal of the American Ceramic Society, 2000, 83(1): 124-128.

[87] BRENNAN R E, TURCU S, HALL A, et al. Fabrication of electroceramic components by layered manufacturing (LM). Ferroelectrics, 2003, 293(1): 3-17.

[88] SAFARI A, ALLAHVERDI M, AKDOGAN E K. Solid freeform fabrication of piezoelectric sensors and actuators. Journal of Materials Science, 2006, 41: 177-198.

[89] SAFARI A, AKDOGAN E K. Rapid prototyping of novel piezoelectric composites. Ferroelectrics, 2006, 331(1): 153-179.

[90] KIM H, FERNANDO T, LI M, et al. Fabrication and characterization of $3 \mathrm{D}$ printed $\mathrm{BaTiO}_{3} / \mathrm{PVDF}$ nanocomposites. Journal of Composite Materials, 2018, 52(2): 197-206. 